\title{
ON THE WEDDLE SURFACE AND ANALOGOUS LOCI*
}

BY

\section{ARNOLD EMCH}

\section{INTRODUCTION}

Geometrically, the well known quartic, the Weddle surface, may be defined as the locus of the vertices of the quadric cones in the linear threedimensional system of quadrics through six independent points in space. In a similar manner, the locus of the vertices of cones through seven points, or through the eight base points of the net of quadrics determined by the seven points, is a sextic of genus three, which however is not the most general sextic with this genus.

It is the purpose of this paper to establish analogous loci for cubic cones, and, subsequently, for general $n$-ic cones. The base points through which the cones pass shall be denoted by $\mathfrak{A}_{1}, \mathfrak{A}_{2}, \ldots, \mathfrak{A}_{i}$, the vertices of the coördinate tetrahedron by $A_{1}, A_{2}, A_{3}, A_{4}$, the join of any two base points $\mathfrak{A}_{i}$ and $\mathfrak{A}_{k}$ by $g_{i k}$ or simply by $g$; a surface locus of vertices of cones by $W$, a curve locus by $S$.

In case of $n$-ic cones there is $i=N+1(N=n(n+3) / 2)$ for a $W$, $i=N+2$, for an $S$.

Before taking up the cubic cones it is perhaps well to state briefly some of the properties of the locus of vertices of quadric cones through six points, and through the eight base points of a net of quadrics.

\section{The Weddle surface}

When $n=2, N+1=6$, the order of $W$ is $m=4$, and we have the Weddle surface as the locus of quadric cones through six points. The number of lines $g$ is 15 which in sets of 5 pass through the six points, the nodes of $W$. The six points can be arranged in 10 double-triples, each of which determines two planes through the six points, or a degenerate cone whose axis lies on $W$. Thus, in addition to the $15 \mathrm{~g}$ 's, there are 10 other lines on $W$, so that the total number of lines on $W$ is 25 , as is well known.

The order of the curve $S$ of vertices of quadric cones through seven points is $s=6$. But in the case of $n=2$ we have the circumstance that all quadric cones through seven points pass through an eighth point, so that the $S_{6}$ appears as the locus of vertices of quadric cones in a net of quadrics.

* Presented to the Society, December 29, 1923. 
The $S_{6}$ cuts each of the 28 lines joining every pair of the base points in 2 points. Every sextuple of the base points determines a Weddle surface, so that there are 28 Weddle surfaces through the $S_{6}$. The $S_{6}$ is also the base curve of the involutory cubic Cremona transformation connected with the eight base points, and the surface of the eighth order formed by the trisecants of the $S_{6}$ is the base surface of the transformation. The cubic surfaces corresponding to any two planes pass through $S_{6}$, and intersect moreover in a cubic rest curve which corresponds to the line of intersection of the two planes. The $S_{6}$ is therefore of genus 3. But this $S_{6}$ is not the most general sextic of this genus, since the system of general sextics of genus 3 has the dimension 24 that of our system being $3 \cdot 7=21$. For 7 points determine such a sextic and there are $\infty^{8.7}$ sextuples. What characterizes our sextic is the fact that it is uniquely determined by the 8 base points of a net of quadrics. The number 21 as the dimension of the system of sextics is also verified as follows. There are $\infty^{18}$ sextuples of points and consequently the same manifold of Weddle surfaces. With a definite Weddle surface fixed, every seventh point determines an $S_{6}$ uniquely. Hence on every Weddle surface there are $\infty^{3}$ sextics, so that their manifold is $\infty^{21}$.

In the involutory cubic transformation determined by the octuple of base points, bisecants of the $S_{6}$ are transformed into bisecants of $S_{6}$, a quartic surface through $S_{6}$ into a quartic which passes singly through $S_{6}$, because every trisecant of $S_{6}$ cuts the original quartic in one point only (outside of $S_{6}$ ). The 28 Weddle surfaces through $S_{6}$ are therefore invariant in the transformation.

Now with every Weddle surface is also uniquely associated a Geiser transformation of order 7. As is well known this involutory Cremona transformation may be defined by the net of quadrics through the six nodes of the surface and a seventh point $P$. Then all quadrics of this net will pass through an eighth point $P^{\prime}$. Thus to every point $P$ corresponds a point $P^{\prime}$ and conversely to $P^{\prime}$ corresponds in the same transformation $P$. The Weddle surface is pointwise invariant in this transformation and passes through a twisted cubic on the six nodes. The join of $P$ and $P^{\prime}$ is a bisecant $s$ of this cubic, so that corresponding pairs on $s$ form an involution with the intersection of $s$ and the Weddle surface (outside of the cubic) as double points. Summing up, we have

TheoRem 1. There are 28 Weddle surfaces through a sixtic $S_{6}$ determined by the octuple of base points of a net of quadrics. Each of the $W$ 's is invariant in the involutory cubic transformation determined by the octuple. There are also 28 involutory Geiser transformations each leaving one of the $W$ 's, and consequently the $S_{6}$, pointwise invariant.

The 28 Geiser transformations determine an infinite discontinuous group $G$ which leaves the sextic pointwise invariant. 
Denoting by $T_{i k}$ the Geiser transformation associated with the Weddle surface $W_{i k}$ which has the six points obtained from the octuple $A_{h}(h=1,2, \cdots, 8)$ by leaving out $A_{i}$ and $A_{k}(h=i, h=k), T_{i k}$ has the property that it permutes $A_{i}$ and $A_{k}$ and leaves the remaining 6 points of the octuple unchanged. Thus the $28 T_{i k}$ 's leave the configuration of the octuple unchanged. Any permutation of the 8 points may be obtained as a product of Geiser transformations $T_{i k}$. For example, $A_{1} A_{2} A_{3} A_{4} A_{5} A_{6} A_{7} A_{8}$ is transformed into $A_{5} A_{3} A_{1} A_{8} A_{4} A_{2} A_{7} A_{6}$ by the transformation $T_{68} T_{56} T_{43} T_{36} T_{26} T_{13}$. This is obvious since every substitution may be obtained as a product of transpositions. The transformations of $G$ therefore transform the octuple in $8 !=40,320$ different ways.

To prove that the transformations of $G$ are infinite in number, consider for example the repeated product $T_{78} T_{12} T_{78} T_{12} \cdots$ and the effect which it has on the Weddle surface $W_{12}$. If the repeated product at some stage could lead to an identity, $W_{12}$, which in turn is transformed by $T_{78}, T_{12}, T_{78}, T_{12}, \ldots$ into surfaces of definite orders, would accordingly be transformed into itself. Now, as a matter of fact, $T_{i 8}$ transforms $W_{12}$ into a surface $W_{12}^{\prime}$ of order 12 with double points at $A_{7}$ and $A_{8}$, six-fold points at $A_{3}, A_{4}, A_{5}, A_{6}$ and eight-fold points at $A_{1}$ and $A_{2}$, as can easily be ascertained. $T_{12}$ transforms $W_{12}^{\prime}$ into a surface $W_{12}^{\prime \prime}$ of order 28 with eight-fold points at $A_{1}$ and $A_{2}$, 14-fold points at $A_{3}, A_{4}, A_{5}, A_{6}, 18$-fold points at $A_{7}, A_{8}$. Next, $T_{73}$ transforms $W_{12}^{\prime \prime}$ into $W_{12}^{\prime \prime}$, of order 52 ; again $T_{12}$ transforms $W_{12}^{\prime \prime \prime}$ into $W_{12}^{(4)}$ of order $84, T_{78}$ transforms $W_{12}^{(4)}$ into $W_{12}^{(5)}$ of order 124 , etc. Thus the succession of $1,2,3,4, \cdots, m, \cdots$ transformations (including 1 as the identical one) leads consecutively to surfaces of order $4,12,28,52,84,124, \cdots, 4\left(m^{3}-m+1\right), \cdots$. From this follows that the repeated products of $T_{78}$ and $T_{12}$ all represent different transformations; and that their number, and hence also the number of transformations of the $G$, is infinite.

\section{W-SURFACE OF VERTICES OF CUBIC CONES}

Let $\mathfrak{A}_{i}\left(a_{i} b_{i} c_{i} d_{i}\right), i=1,2, \cdots, 10$, be the ten base points and $P\left(x_{1}, x_{2}, x_{3}, x_{4}\right)$ any point in space distinct from any of the $\mathfrak{A}_{i}$ 's. Assume the coördinate tetrahedron in a general position, and project the points $\mathfrak{A}_{i}$ from $P$ upon $x_{4}=0$. The coördinates of the projections $\mathfrak{A}_{i}^{\prime}$ are

$$
\varrho X_{i}=a_{i} x_{4}-d_{i} x_{1}, \quad \varrho Y_{i}=b_{i} x_{4}-d_{i} x_{2}, \quad \varrho Z_{i}=c_{i} x_{4}-d_{i} x_{3},
$$

or, writing cartesian coördinates in space

$$
x=\frac{x_{1}}{x_{4}}, \quad y=\frac{x_{2}}{x_{4}}, \quad z=\frac{z_{3}}{z_{4}},
$$


and putting $d_{i}=1$,

$$
\sigma X_{i}=x-a_{i}, \quad \sigma Y_{i}=y-b_{i}, \quad \sigma Z_{i}=z-c_{i} .
$$

The ten projections $\mathfrak{A}_{i}^{\prime}$ lie on a cubic in $x_{4} \leq 0$, when the determinant

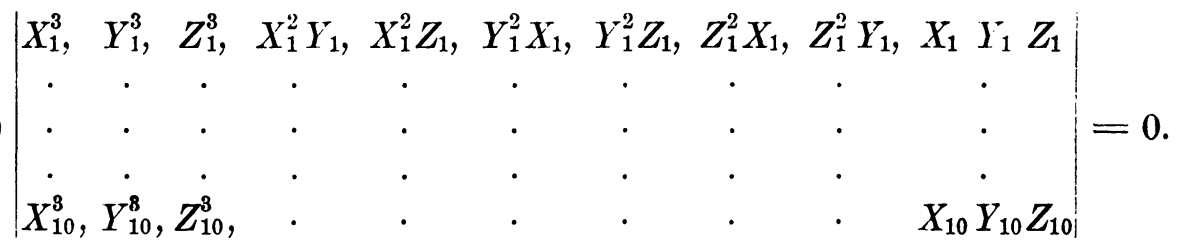

The expansion of this gives an equation of degree 30 in $x, y, z$ for the locus of vertices $(x, y, z)$ of cubic cones through the ten given points. But this equation is reducible, as is seen by expanding the $X, Y$, and $Z$ terms in (2), and by subtracting the first row in succession from the 9 remaining rows. In this manner all cubic terms in the 9 rows vanish, so that the degree of the expanded deterninant now becomes 21. By repeated division by proper numerical factors and subscquent subtraction the degree may be reduced still further. But the degree of the final irreducible equation may be established by a much less laborious method. The order of the $W$-surface is equal to the number of its intersections with a generic line. Such a line is $y=0, z=0$. Substituting this in (2) the first column contains only cubic terms in $x$, the second and third columns only constants, the fourth and fifth only quadratic terms in $x$, the sixth only linear terms in $x$, the seventh constant terms, the eighth linear terms in $x$, the ninth constant terms, and the tenth linear terms in $x$. The determinant consequently expands into a polynomial of degree $1 \cdot 3+2(3-1)+3(3-2)=10$ in $x$. Consequently the line $y=0, z=0$ cuts the $W$-surface in 10 points. When the determinant (2) is expanded by multiplying the elements of the $k$ th row by their corresponding minors, a homogeneous polynomial of degree 3 in $x-a_{k}, y-b_{k}, z-c_{k}$ is obtained. From this follows that the point $\left(a_{k}, b_{k}, c_{k}\right)$ is a triple point of the surface, and that the cubic cone with this point as a vertex, and through the remaining nine points, is the tangential cone to the surface at this point.

Consider any point $V$ of a line $g$ joining any two of the ten points $\mathfrak{A}_{i}$. This line $g$ and the 9 lines joining $V$ to the 8 remaining points determine a cubic cone uniquely. This is a cubic cone through the 10 points, consequently $V$, and the line $g$, lie on the $W$-surface. These results may be summed up in

THEOREm 2. The locus of vertices of cubic cones through 10 fixed points in space is a surface of order 10 with these points as triple-points and with the 45 joins of the 10 points as single lines on the surface. 


\section{IV. $S$-CuRve OF Vertices OF CUBIC CONES}

In addition to the 10 fixed points $\mathfrak{A}_{1}, \ldots, \mathfrak{A}_{10}$ of the $W$-surface of order 10 consider another fixed point $\mathfrak{A}_{11}$. The locus of the vertices of cubic cones through these 11 points is a curve $S$ which may be obtained from the intersection of any two $W$-surfaces associated with two sets of ten points each chosen from the 11 points. Thus, $S$ may be obtained from the intersection of the two surfaces $W_{10}$ and $W_{11}$ associated with $\mathfrak{A}_{1} \ldots \mathfrak{A}_{9} \mathfrak{A}_{10}$ and $\mathfrak{A}_{1} \ldots \mathfrak{A}_{9} \mathfrak{A}_{11}$, respectively. Now consider the line $l$ joining $\mathfrak{A}_{10}$ and $\mathfrak{A}_{11}$; it will cut $W_{10}$ in 7 points $V_{1}, \ldots, V_{7}$ outside of $\mathfrak{U}_{10}$ which is a triple point of $W_{10}$. Hence $V_{1}, \cdots, V_{7}$ are vertices of cubic cones which pass through all 11 points and are therefore points of $S$. Thus, each of the 55 joins $l$ of the 11 points cuts the curve $S$ in 7 points. $S$ itself does not pass through the 11 points $\mathfrak{A}_{1}, \cdots, \mathfrak{A}_{11}$. This fact may be immediately verified analytically by choosing $\mathfrak{A}_{10}$ at $\left(a_{10}=0, b_{10}=0, c_{10}=0\right)$, and $y=0, z=0$ as the line $l$. Then (2) reduces to an equation of degree 7 in $x$.

As $W_{10}$ and $W_{11}$ have the 36 joins $g$ of $\mathfrak{A}_{1}, \ldots, \mathfrak{A}_{9}$ in common, their curve of intersection outside of these lines is of order 64 . But it is reducible. To show this take the plane $p$ determined by any triple of points out of the nine common to $W_{10}$ and $W_{11}$, say by $\mathfrak{A}_{1}, \mathfrak{A}_{2}, \mathfrak{A}_{3}$. Let $g_{1}, g_{2}, g_{3}$ be the joins of $\mathfrak{A}_{2} \mathfrak{A}_{3}, \mathfrak{A}_{3} \mathfrak{A}_{1}, \mathfrak{A}_{1} \mathfrak{A}_{2}$, and $B_{1}, B_{2}, \cdots, B_{15}$ the intersections with $p$ of the 15 joins $g$ of $\mathfrak{A}_{4}, \mathfrak{A}_{5}, \ldots, \mathfrak{A}_{9}$. The plane $p$ cuts $W_{10}$ and $W_{11}$ each in $g_{1}, g_{2}, g_{3}$ and a curve of order 7 which passes through the $15 B$ 's and singly through $\mathfrak{A}_{1}, \mathfrak{A}_{2}, \mathfrak{A}_{3}$, since these are triple points. Hence the two curves of order 7, outside of the $B$ 's and $\mathfrak{X}$ 's intersect in $49-15-3=31$ residual points, which lie on the curve $S$. But $S$ cuts each $g_{1}, g_{2}, g_{3}$ in 7 points. Consequently $p$ intersects $S$ in $31+21=52$ points, which is the order of $S$.

As the curve of intersections of $W_{10}$ and $W_{11}$ outside of the $36 \mathrm{~g}$ 's is of order 64, of which $S$ is a part, there is a residual curve $R$ of order 12 . Since each of the $9 \mathfrak{A}$ 's common to $W_{10}$ and $W_{11}$ is a triple point for both surfaces at which $8 \mathrm{~g}$ 's, common to $W_{10}$ and $W_{11}$, are concurrent, the $R$ passes through each of the 9 points. Thus, in the plane $p, R$ passes singly through $\mathfrak{A}_{1}, \mathfrak{A}_{2}, \mathfrak{A}_{3}$, and must cut $g_{1}, g_{2}, g_{3}$ each in 3 additional points. The curve $R$ does not belong to the locus of vertices of cubic cones through 11 points. To ascertain the geometric meaning of $R$, consider a pencil $\Phi_{1}+\lambda \Phi_{2}=0$ of cubic cones through 8 of the 9 points, say $\mathfrak{A}_{1}, \cdots, \mathfrak{A}_{8}$, with $V(x, y, z)$ as a vertex. All cones of the pencil pass through the 8 lines $V \mathfrak{A}_{1}, \cdots, V \mathfrak{A}_{8}$, and a ninth fixed line $g_{9}$. Intersect this pencil with $x_{1}=0$, as before, and denote the projections by the same letters with accents. Then in order that $\mathfrak{A}_{9}^{\prime}$ may coincide with the intersections of $g_{9}$ with $x_{4}=0$, on substituting the coördinates of $\mathfrak{A}_{9}$ in 
$\Phi_{1}^{\prime}+\lambda \Phi_{2}^{\prime}=0$, there must be simultaneously $\Phi_{1}^{\prime}=0, \Phi_{2}^{\prime}=0$. The irreducible part of the intersection of the two surfaces is precisely the curve $R$. From every point of $R$ the nine points are projected into 9 points of a pencil of cubics. Hence there is a cubic of the pencil through $\mathfrak{A}_{10}^{\prime}$ and another cubic through $\mathfrak{A}_{11}^{\prime}$. Consequently every point of $R$ is a vertex of two cubic cones through $\mathfrak{A}_{1}, \ldots, \mathfrak{A}_{\theta}, \mathfrak{A}_{10}$ and $\mathfrak{A}_{1}, \ldots, \mathfrak{A}_{\theta}, \mathfrak{A}_{11}$. The result may be stated in

THEOREM 3. The locus of vertices of cubic cones through 11 points is a curve $S$ of order 52, which cuts each of the 55 joins in 7 points.

There are $55 \mathrm{~W}$-surfaces through $S$. Any two surfaces of the set intersect in a residual curve $R$ of order 12 , uniquely associated with the 9 points $\mathfrak{A}$ common to the two $W$ 's. The curve $R$ is the locus of points from which the nine points are projected into the base points of a pencil of cubics.

The curve $S$ has no effective singularities, so that its genus may be determined by evaluating the number of its apparent double points from any generic point $O$, and by subtracting this number from the maximum number of double points of its projection. The sextic cone $C$ through $S$ with $O$ as a vertex cuts $W_{11}$, outside of $S$, in a residual curve $S^{*}$ of order $10 \cdot 52-52=468$. Now $S^{\prime}$ cuts $W_{10}$ in 4680 points. Among these points are the $9.52=468$ points in which the first polar of $O$ with respect to $W_{11}$ cuts $S$. At such a point the tangents to $S$ and $S^{\prime}$ at this point lie in the tangent plane to $W_{11}$ at this point. Hence the projection of a point does not result in a double point. The cone $C$ cuts each join $g$ of the nine points $\mathfrak{A}$, associated with $S$, in 52 points, among which are the 7 intersections of $g$ with $S$, which do not lie on $S^{*}$. The remaining 45 points on each $g$ lie on $S^{*}$, and on $W_{10}$, but not on $S$. Thus there are $36 \cdot 45=1620$ such points, which also are not projected into double points of the projections of $S^{\prime}$ of $S$ from $O$. The remaining 4680-468 $-1620=2592$ intersections of $S^{*}$ with $W_{10}$ arrange themselves into 1296 couples $P, P^{\prime}$ which lie similtaneously on $S$ and $S^{*}$ and on lines through $O$. In fact, such a point of intersection $P^{*}$ of $S^{*}$ with $W_{10}$ lies on $W_{11}$ and $W_{10}$, and consequently on $S$. But $P^{*}$ is the projection from $O$ upon $W_{11}$ of a point $P$ and $S$, so that $P^{*}$ is projected back into $P$, and $P$ also lies on $S^{*}$.

The genus of $S$ is therefore $\frac{1}{3} \cdot 51 \cdot 50-1296=1254$.

As a verification, the same method applied to the curve $S$ in case of the Weddle surface yields the genus 3, as is well known.

\section{V. $W$-SURFACES OF VERTICES OF $n$-IC CONES}

Following the same method and notation as in the case of cubic cones it is easy to generalize the results for $n$-ic cones, and it is not necessary to restate the arguments in detail. 
The determinant (2) is now of order $N+1$ and expands into a polynomial of degree $n(N+1)$ in $x, y, z$. If we write the equation of the $n$-ic in $x_{4}=0$ in the form

$$
X^{n}+X^{n-1} \Phi_{1}+X^{n-2} \Phi_{2}+\cdots+\Phi_{n}=0,
$$

in which $\boldsymbol{\Phi}_{i}$ is a homogeneus polynomial of degree $i$ in $Y$ and $Z$, so that $\boldsymbol{\Phi}_{i}$ contains $i+1$ terms, the determinant

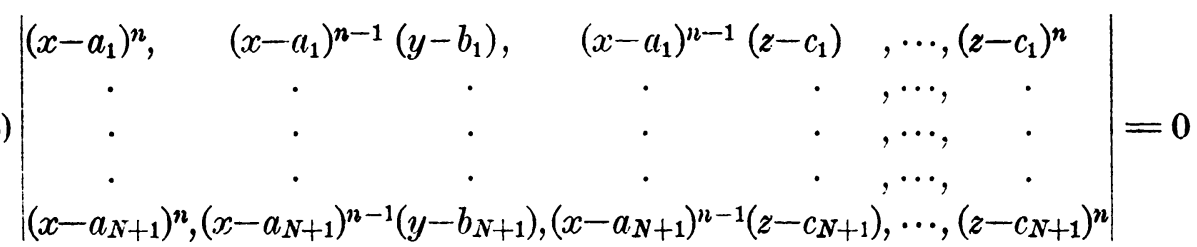

contains $x^{n}$ as the highest power in the first column only, $x^{n-1}$ in the next two, $x^{n-2}$ in the next three, $x^{n-k}$ in $k+1$ consecutive columns. The reduced form of (3) gives the equation of the $W$-surface. It is evident from (3) that the generic line $y=0, z=0$ gives for its intersection with $W$ an equation of degree

(4) $m=1 \cdot n+2(n-1)+3(n-2)+\cdots+(n-1) 2+n \cdot 1$

$$
=\frac{n(n+1)(n+2)}{6}
$$

in $x$. Hence $m$ is the order of the $W$-surface. Moreover if we write (3) in the form (2) and expand from the elements of the $k$ th row, it is apparent that $\mathfrak{A}_{k}$ is an $n$-fold point of $W$. Again, every point of a join $g$ of two of the $N+1$ points is a vertex of an $n$-ic cone through the $N+1$ points. Hence

TheOREm 4. The locus of vertices of $n$-ic cones through $\frac{1}{2} n(n+3)+1$ points $\mathfrak{A}_{i}$ is a $W$-surface of order $\frac{1}{6} n(n+1)(n+2)$ with the $\mathfrak{A}_{i}$ 's as $n$-fold points and the joins $g$ as single lines. The tangential cones at the $\mathfrak{A}_{i}$ 's are determined by the $N$ joins $g$ through each $\mathfrak{A}_{i}$.

\section{VI. $S$-Curves}

The locus of vertices of $n$-ic cones through $N+2$ points is an $S$-curve, through which all the $\frac{1}{2}(N+2)(N+1) W$-surfaces associated with the $(N+1)$-tuples of the $N+2$ points pass. Let $W_{N+1}$ and $W_{N+2}$ be two of these surfaces with the group of common points $(\mathfrak{U})=\left(\mathfrak{A}_{1}, \mathfrak{A}_{2}, \cdots, \mathfrak{A}_{N}\right)$ and with $\mathfrak{A}_{N+1}$ and $\mathfrak{A}_{N+2}$ individualizing the two surfaces respectively. Their full intersection, to which $S$ belongs, is of order $m^{2}=\frac{1}{36} n^{2}(n+1)^{2}(n+2)^{2}$, 
from which split off the $\frac{1}{2} N(N-1)$ joins $g$ of $(\mathfrak{A})$. The intersection proper of $W_{N+1}$ and $W_{N+2}$ is therefore a curve $T$ of order

$t==m^{2}-\frac{1}{2} N(N-1)=\frac{1}{72}\left(2 n^{6}+12 n^{6}+17 n^{4}-30 n^{3}-55 n^{2}+54 n\right)$.

This curve is composed of $S$ and a residual curve $R$. To determine the order of these curves, the number of points in which $S$ cuts a join $g$ must first be established. Let $g$ be the join of $\mathfrak{A}_{N+1}$ and $\mathfrak{A}_{N+2}$. It cuts $W_{N+1}$ in $m-n$ points $V$, outside of $\mathfrak{A}_{N+1}$. The $n$-ic cones with the $V$ 's as vertices and through $(\mathfrak{U})$ and $\mathfrak{A}_{N+1}$ also pass through $\mathfrak{U}_{N+2}$; consequently the same $V$ 's are also vertices of $n$-ic cones through $(\mathfrak{A})$ and $\mathfrak{A}_{N+2}$, and lie on $W_{N+2}$, and hence on $S$. Thus, $S$ cuts each $g$ in $m-n$ points. Next cut both $W_{N+1}$ and $W_{N+2}$ by the plane $p$ through $\mathfrak{A}_{1}, \mathfrak{A}_{2}, \mathfrak{A}_{3}$. Neglecting the joins $g_{1}, g_{2}, g_{3}$, which are common to both surfaces, the residual insersections are curves $C_{1}$ and $C_{\mathrm{z}}$ of order $m-3$, which both pass through the $\frac{1}{2} N(N-1)-3$ intersections $B$ of the joins $g$ of the group ( $\mathfrak{X}$ ) (minus the three lines $\left.g_{1}, g_{2}, g_{3}\right)$. As $W_{N+1}$ and $W_{N+2}$ have $n$-tuple points at $\mathfrak{A}_{1}, \mathfrak{A}_{2}, \mathfrak{A}_{3}$, and at each of these have $N-1 \mathrm{~g}$ 's in common, the curve $T$ has multiplicities of order $n^{2}-N+1=\frac{1}{2}(n-1)(n-2)$ at each $\mathfrak{A}_{1}, \mathfrak{A}_{2}, \mathfrak{A}_{3}$. The multiplicity of each $C_{1}$ and $C_{2}$ at these points is $n-2$. Hence these points and the points $B$ absorb $\frac{1}{2} N(N-1)-3+3(n-2)^{2}$ of the $(m-3)^{2}$ intersections of $C_{1}$ and $C_{2}$. The residual set of intersections, that of $S$ with $p$, outside of $g_{1}, g_{2}, y_{3}$, consists therefore of

$$
\begin{aligned}
\mu & =(m-3)^{2}-\frac{1}{2}(N-3)(N-4)-3(n-2)^{2} \\
& =\frac{1}{72}\left(2 n^{6}+12 n^{5}+17 n^{4}-102 n^{3}-379 n^{2}+1098 n-648\right) .
\end{aligned}
$$

To this number must be added the $3(m-n)=\frac{1}{2}\left(n^{3}+3 n^{2}-4 n\right)$ intersections of $S$ with $g_{1}, g_{2}, g_{3}$. Hence the order of $S$ is

$$
s=\frac{1}{72}\left(2 n^{6}+12 n^{5}+17 n^{4}-66 n^{3}-271 n^{2}+954 n-648\right) .
$$

The order of $R$, the residual curve of intersection of $W_{N+1}$ and $W_{N+2}$, is $r=t-s$, or

$$
r=\frac{1}{2}\left(n^{3}+6 n^{2}-25 n+18\right)=\frac{1}{2}(n-1)(n-2)(n+9) .
$$

$R$ is the locus of points from which the $N$ joins of the group ( $\mathfrak{A}$ ) are projected into the base points of a pencil of $n$-ics. 
Thus may be stated

Theorem 5. The locus of vertices of $n$-ic cones through $\frac{1}{2} n(n+3)+2$ points $\mathfrak{A}$ in space is an S-curve of order

$$
s=\frac{1}{72}\left(2 n^{6}+12 n^{5}+17 n^{4}-66 n^{3}-271 n^{2}+954 n-648\right),
$$

which cuts each of the $\frac{1}{2}(N+1)(N+2)$ joins of each two of the $\mathfrak{A}$ 's in $\frac{1}{2} n(n+1)(n+2)-n$ points, and does not pass through the $\mathfrak{A}$ 's. Any two $W$-surfaces each associated with $N+1$ points of the set $\mathfrak{A}$ pass through $S$ und intersect moreover in a residual curve $R$ of order

$$
r=\frac{1}{2}(n-1)(n-2)(n+9)
$$

which has multiple points of order $\frac{1}{2}(n-1)(n-2)$ at the points of the group (II) associated with $R . R$ is the locus of points from which the group (趾 is projected into the base of a pencil of $n$-ics.

The genus of $S$ (and also of $R$ ) could easily be determined as in the case of cubic cones and, for the sake of brevity, will be omitted.

UNIVRRSITY OF ILIINOIS,

URBANA, IIJ.. 